\title{
In-Field Demonstration of a Photonic Coherent MIMO Distributed Radar Network
}

\author{
Leonardo Lembo \\ TECIP-Vallauri Institute \\ Scuola Superiore Sant'Anna-CSSN \\ Pisa - Italy \\ leonardo.lembo@sssup.it
Filippo Scotti
PNTLab
CNIT
Pisa - Italy \\ filippo.scotti@cnit.it
}

\author{
Salvatore Maresca \\ TECIP Institute \\ Scuola Superiore Sant'Anna \\ Pisa - Italy \\ salvatore.maresca@sssup.it
}

\author{
Giovanni Serafino \\ TECIP Institute \\ Scuola Superiore Sant'Anna \\ Pisa - Italy \\ giovanni.serafino@sssup.it
}

\author{
Francesco Amato \\ TECIP Institute \\ Scuola Sant'Anna \\ Pisa - Italy \\ francesco.amato@sssup.it
}

\author{
Paolo Ghelfi \\ PNTLab \\ CNIT \\ Pisa - Italy \\ paolo.ghelfi@cnit.it
}

\author{
Antonella Bogoni \\ TECIP Institute \\ Scuola Sant'Anna \\ Pisa - Italy \\ antonella.bogoni@sssup.it
}

\begin{abstract}
This paper reports an in-field experiment of a photonics-based coherent MIMO radar network. The use of photonics guarantees the coherence of the transmitted and received RF signals, and allows remoting the antennas exploiting deployed optical fibers, thus a MIMO approach can be applied on a network of widely distributed coherent radars. In the infield experiment, a photonics-based radar core connects two transmitters and two receivers, with $100-\mathrm{MHz}$ bandwidth signals in $\mathrm{X}$-band, observing a collaborative target. The results demonstrate an improvement in radar precision, and envisage real applications wherever fiber is available for deploying the radar network.
\end{abstract}

Keywords-MIMO radar; Multi-static radar; Distributed radar network; Photonic Coherent radar network.

\section{INTRODUCTION}

The pace of the technological advances in the communications field is underpinning the unrestrainable increment of worldwide data stream. In fact, the maximum transmission capacity in communication systems has been increasing with an impressive annual rate [1] in order to satisfy the ever-growing customer demand. Despite this trend, information sharing in the specific case of radar systems is rather limited: in fact, if multiple radar systems are present in the same area or on board of the same platform (e.g., a ship), they generally do not co-operate but rather provide multiple uncorrelated information of the same scene.

The paradigm of multiple radars is often implemented in the form of multistatic radars, i.e. radars with multiple transmitters and/or receivers positioned in different places. These multiple radars act independently or with poor coordination, and they exchange only a limited amount of data. In fact, each node of the multistatic radar network performs a local pre-processing (e.g., detection, tracking), and then sends the results to a master node for data fusion. On one hand, this solution avoids any issue related to the synchronization of all the network nodes, and reduces the amount of data to be shared. On the other hand, the tribute to pay for this simplification is an evident performance limitation determined by the fact that data fusion is done on already elaborated plots and not directly on the acquired samples of each signal.

Recently, multiple radars with a higher degree of coordination have being proposed, where the transmitters make use of different waveforms, so that each receiver can process signals coming from multiple distributed emitters. These multiple radars are usually named multiple-input multiple-output (MIMO) radars [2]. In MIMO radars, the increased spatial information of the distributed radar network improves the overall detection performance, in particular when the target exhibits a low radar cross section (RCS), or when the RCS is characterized by a high angular variability. Furthermore, MIMO radars allow achieving higher crossrange resolution than the one given by the signal bandwidth (BW).

In [3],[4], an innovative radar prototype has been presented and successfully tested for the first time, both in maritime and in air monitoring scenarios. The peculiarity of this radar is that the signal generation, distribution and processing are based on photonics, and this approach guarantees an unprecedented frequency flexibility and phase noise stability. Additionally, the radar signal distribution through optical fibers assures high-quality and highcoherence, as well as large BW connectivity between the radars. Therefore, photonics can become an enabling technology for the realization of MIMO radar networks with widely distributed antennas. In fact, a distributed coherent photonic radar network can be imagined being composed of a central photonic core, capable to distribute optical signals via optical fiber to multiple antennas, where a conversion from optical to RF domain is operated before the RF front end. Conversely, the RF echoes received from the targets are locally converted at the antennas from the RF to the photonics domain, and sent back to the central processing core for processing. Recently, in [5] the first photonic MIMO radar has been tested in a controlled environment with a signal having a BW of $4 \mathrm{GHz}$ in the K-band. Thanks to the MIMO processing, it has been possible locate targets with a precision of $1.5 \mathrm{~cm}$.

Exploiting the design indications emerged from the analysis described in [6], in this paper we report on the first coherent photonics-based MIMO radar network tested in a real out-door scenario. The implemented MIMO radar network is composed of two transmitters and two receivers connected to a photonic core through optical fibers. The experiment is carried out adopting setup parameters suitable for coastal surveillance application. In particular, a $100 \mathrm{MHz}$ BW chirp signal in X-band is used, while signal orthogonality between the two transmitters is achieved adopting a time domain multiplexing (TDM) approach. Although in our experiment the four antennas are distributed along a baseline of few tens 


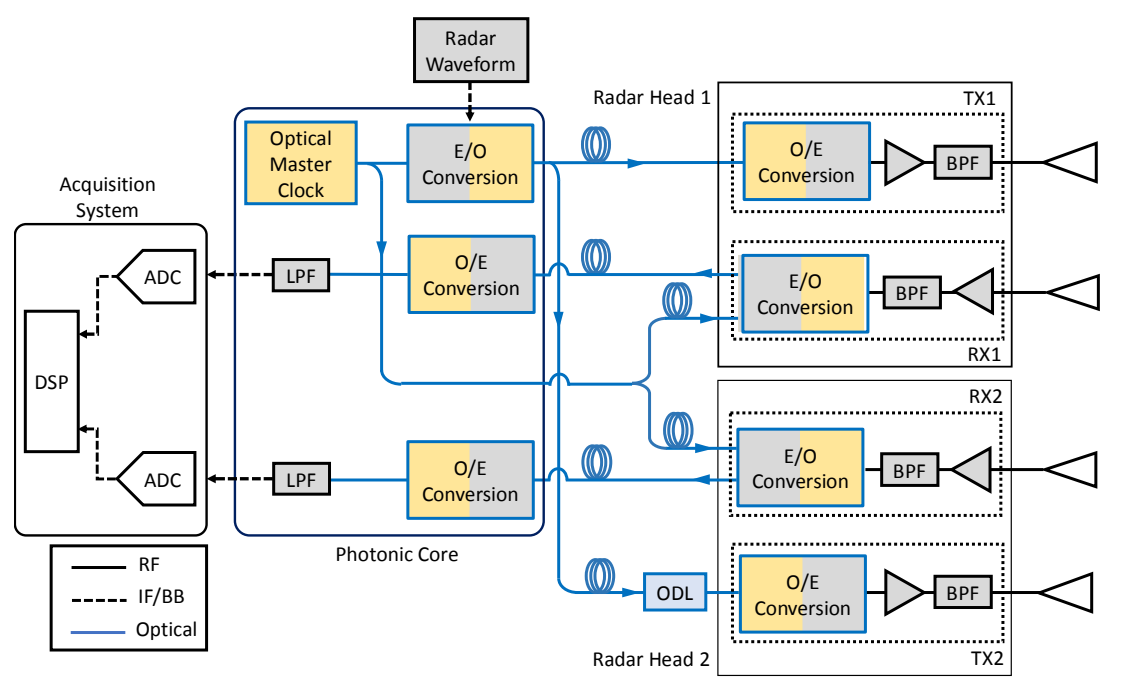

Fig. 1: Photonic Radar Network Architecture. DSP: Digital Signal Processing; ADC: Analog-to-Digital Converter; RF: Radio Frequency; LPF: Low-Pass Filter E/O: Electro-Optical; ODL: Optical Delay Line; O/E: Opto-Electrical; IF/BB: Intermediate Frequency/Base Band; BPF: Band-Pass Filter; TX: Transmitter; RX: Receiver.

of meters, the long spools of fiber inserted in the setup emulate a baseline of about $1 \mathrm{~km}$. The target, which consists in a small metallic cylinder carried by a mini-drone, is correctly detected with the expected precision, confirming the validity of the proposed architecture. Although the dataset is affected by all typical phenomena present in a real environment, the superiority of a coherent MIMO processing compared with a non-coherent processing approach is apparent. With respect to previously published fiber-distributed MIMO radars [5],[7], in this work we make use of much narrower signal BW, though achieving a fine precision thanks to the coherent MIMO processing.

This paper is organized as follows: Section II is dedicated to the description of the photonic architecture; in Section III, the algorithms adopted for the data set processing are introduced, and in Section IV the experimental setup is discussed. In Section V, the results obtained applying a noncoherent processing and a MIMO algorithm are discussed and compared. Finally, Section VI summarizes the work and draws the conclusions.

\section{DEMONSTRATOR ARCHITECTURE}

The demonstrator of the photonic distributed radar is composed of a photonic core, a co-located acquisition system, and two remoted radar heads (RHs). Each RH is divided into one transmitter (TX) and one receiver (RX). The architecture of the demonstrator is schematically depicted in Fig. 1.

The photonic core contains the optical master clock, implemented by a solid-state mode-locked laser (MLL), which can be considered as a multi-frequency optical oscillator. Indeed, the MLL is a pulsed laser; therefore its optical spectrum is a comb of several lines, spaced by the pulses repetition frequency, which in this case is $400 \mathrm{MHz}$. The fundamental feature of this kind of laser is the finest phase locking of the lines, which allows the generation of RF carriers with extremely low phase noise, upon the opto-electronic $(\mathrm{O} / \mathrm{E})$ conversion of the MLL laser in a photodiode [8]. This feature permits the high-quality implementation of both an RF up-conversion and an RF down-conversion. In the case of upconversion, the radar signal digitally generated at intermediate frequency (IF) is first modulated on the MLL spectrum in an electro-optic modulator, and then up-converted to each multiple frequency of the MLL repetition rate [3],[4],[9]. The desired output RF frequency can be selected by means of a microwave filter. In the case of down-conversion, the signal at RF is similarly modulated on the MLL, and then the O/E conversion at the photodiode generates replicas of the RF signal down-converted at any multiple of the MLL repetition rate, including the IF closest to baseband [3],[11]. The large optical spectrum of the MLL ensures that these operations are highly efficient, and the wide $\mathrm{E} / \mathrm{O}$ and $\mathrm{O} / \mathrm{E}$ bandwidth of the available devices (respectively, modulators and photodiodes) permits the flexible management of RF signals up to several tens of GHz.

As reported in Fig. 1, the optical master clock is split into two arms. One arm is devoted to the $\mathrm{E} / \mathrm{O}$ conversion of the radar waveform in transmission, and is then connected to the TXs at the RHs. The other arm instead is directly connected to the RXs in the RHs. The connections between the photonic core and the TXs and RXs at the RHs are realized through spans of single-mode fiber (SMF), represented by the coils sketched in Fig. 1. This optical distribution of the radar signals over fiber has the advantages of small propagation losses, absence of electromagnetic interference, and preservation of the signal coherence even with large BW [10].

Once the E/O converted radar waveform is delivered to a $\mathrm{TX}$, this operates its opto-electric $(\mathrm{O} / \mathrm{E})$ conversion implying the up-conversion of the IF signal to the RF carrier. Here, the radar signal is generated at $\mathrm{IF}=100 \mathrm{MHz}$, and the RF is selected at $9.7 \mathrm{GHz}$. Thus, after proper amplification and band-pass filtering, the radar signal is launched by the antenna. The employed band-pass filters (BPFs) are multi-cavity filters with a $3-\mathrm{dB} \mathrm{BW}$ of $100 \mathrm{MHz}$ centered at $9.7 \mathrm{GHz}$. The employed antennas at the TXs are ultra-wideband $(1-18 \mathrm{GHz})$ Vivaldi-shaped horn antennas with $50^{\circ}$ half power beam width (HPBM) aperture and 12-dB gain. It is worth underlining the presence of an optical delay line (ODL) before the TX of RH2, to implement the TDM of the two RHs. The ODL is realized by means of a $1 \mathrm{~km}$-long spool of optical fiber. Considering the SMF core refractive index and the related propagation speed of light, this corresponds to a delay $\Delta \mathrm{T}=5 \mu \mathrm{s}$, which introduces a time-orthogonality between the waveforms transmitted by RH1 and RH2. The presence of the ODL also emulates the effect of placing the radar heads along a largely distributed baseline. 


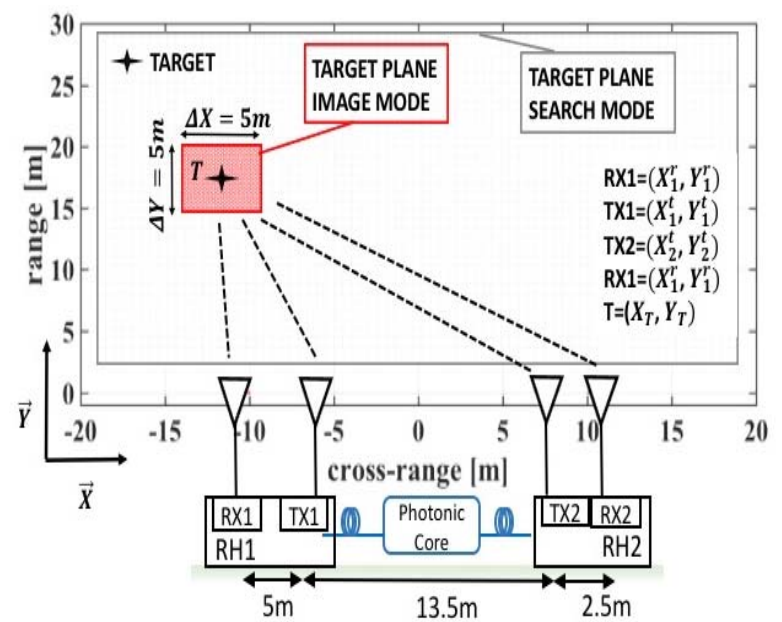

Fig. 2: Distributed coherent photonic radar geometry and experimental setup.

The RXs are equipped with antennas similar to those of the TXs, only with slightly different size, exhibiting a HPBM of $46^{\circ}$ and $12-\mathrm{dB}$ gain, operating in the frequency range $2-18$ GHz.. The detected radar echoes received by the RHs antennas are amplified, pass-band filtered and E/O converted, i.e., modulated on the MLL signal arriving from the photonic core. Then, the received signals are transmitted back to the photonic core, and re-translated into the electrical domain by $\mathrm{O} / \mathrm{E}$ conversion. After this operation, the signal from each $\mathrm{RX}$ is low-pass filtered and fed into the acquisition system, where it is digitized by an analog-to-digital converter (ADC) at $400 \mathrm{MS} / \mathrm{s}$, and eventually processed by the digital signal processing (DSP), which will be described in the following sections.

In our experiment, the employed radar waveform is a rectangular pulse with a duration of $100 \mathrm{~ns}$ and a pulse repetition interval (PRI) of $50 \mu \mathrm{s}$, corresponding to a pulse repetition frequency (PRF) of $20 \mathrm{kHz}$ and $7.5 \mathrm{~km}$ of unambiguous range. The pulse frequency is linearly chirped over $100 \mathrm{MHz}$ around the 9.7-GHz RF carrier, thus allowing a nominal range resolution of $1.5 \mathrm{~m}$.

It is worth to underline here that exploiting a single MLL for all the operations of up- and down-conversion ensures a perfect synchronization of the RHs and maintains the coherence of the involved signals. These features are fundamental for realizing a coherent distributed radar network, and allows the implementation of a specific MIMO processing, as detailed in the following Sections.

\section{Processing SCHEME}

The dataset acquired by deploying the photonics-based radar network setup has been processed following the general sketch proposed in [12] and [13]. Thus, signal processing can be divided into the following main sets of operations: i) signal conditioning \& decentralized non-coherent processing, with the aim of a preliminary low-resolution detection of targets, and ii) centralized coherent processing, whose purpose is instead the target localization with maximum precision. In Section III-A, the signal conditioning and the decentralized non-coherent processing are presented. This latter procedure will be also referred to as "Search Mode". In Section III-B, the centralized coherent MIMO approach is presented, which will be referred to as "Image Mode". In both Section III-A and III-
$\mathrm{B}$, the processes are described in the general case of a radar network including $M$ transmitters and $N$ receivers.

As pointed out in [6], a MIMO coherent processing requires a heavy computational effort, therefore it is opportune to perform this kind of analysis exclusively on those areas of interest where targets have been pre-detected, as shown in Fig. 2. Limiting the size of the regions of interest, it is possible to apply MIMO coherent processing with a resolution cell size reduced to values down to the carrier wavelength, which are appropriate to resolve multiple closely-spaced targets. In case of small targets (i.e., targets smaller than the radar range resolution capability), it is possible to achieve a more precise target localization compared to standard multistatic decentralized non-coherent processing techniques.

It is worth to underline that, instead of a MIMO noncoherent approach, we have preferred a "decentralized noncoherent processing" procedure, which is described in Section III-A. This solution represents a sub-optimum variant to MIMO non-coherent processing. The received data undergo first "local" detection. Then, such local detections are fused, mimicking the output of a "decentralized" radar network. It is important to observe that, although this solution is not meant to fully exploit the photonic radar network capabilities in terms of phase coherence and centralized processing, at the actual level of system advancement, it can provide us further insights on system detection and localization capabilities, in order to better manage clutter effects while keeping the processing complexity manageable.

In Fig. 2, the different areas of interest for Search and Image modes are represented. The RHs are aligned along a straight line. As indicated, both the processing modes adopt a common two-dimensional Cartesian reference system whose center is at the centre of the array baseline, with the $x$-axis oriented towards the second transmitter, and the $y$-axis perpendicular to the ground plane. Thus, the $x$ coordinate indicates the relative position with respect to the center of the baseline, while the $y$ coordinate indicates the target height above the ground.

\section{A. Search Mode: Signal conditioning \& Decentralized non- coherent processing}

Considering the general case of a radar network comprising $M$ transmitters and $N$ receivers, the photonic core shall have $N$ ADCs digitizing the signals received by the RXs, each of these signals being the combination of the $M$ echoes from each of the TXs, down-converted at IF. In the following, we will indicate with the subscript $k, l$ the generic bistatic pair (also referred to as bistatic channel), i.e., the signal determined by the $k^{\text {th }} \mathrm{TX}$ and $l^{\text {th }} \mathrm{RX}$, for $k=1, \ldots, M$ and $l=1, \ldots, N$.

In the signal conditioning stage, the $N$ digitized data streams are split into the individual bistatic channels for data processing. To maximize the signal-to-noise ratio (SNR), the $\mathrm{M} \times \mathrm{N}$ channels are filtered at IF and then down-converted to base-band (BB). Pulse compression (i.e., matched filtering) is performed on the linearly chirped received pulsed signals. Finally, Doppler processing is applied, and each TX/RX channel undergoes moving target indicator (MTI) filtering for stationary clutter cancellation. For the sake of brevity, we omit to detail these operations, that can be found in [12].

A cell-averaging constant false alarm rate (CA-CFAR) detection strategy is applied to the output of the MTI filter [12], [14], [15]. Let us denote respectively with $\tau_{i}$ and $f_{j}$ the 
generic delay and Doppler frequency indices of the cell-undertest (CUT). Data samples from the CUT, namely the $Y_{i, j}$ detection statistic, are compared against a proper detection threshold $\Lambda_{\mathrm{i}, \mathrm{j}}$, computed from a finite reference window surrounding the CUT, with dimensions $\mathrm{K}_{\mathrm{T}}$ and $\mathrm{K}_{\mathrm{f}}$ along delay and Doppler frequency, respectively. In addition, in the same way $\mathrm{N}_{\mathrm{G}, \tau}$ and $\mathrm{N}_{\mathrm{G}, \mathrm{f}}$ guard cells chosen adjacent to the CUT are not considered in the threshold calculation. This is done to reduce the so-called "target-masking" issue, i.e., the possible leaking of target sidelobes into training cells. Thus, the number of useful delay-Doppler frequency training cells is $\mathrm{K}=\left(\mathrm{K}_{\tau}+\mathrm{N}_{\mathrm{G}, \tau}+1\right) \times\left(\mathrm{K}_{\mathrm{f}}+\mathrm{N}_{\mathrm{G}, \mathrm{f}}+1\right)$. Finally, the statistic is compared with the threshold such that:

$$
Y_{i, j} \triangleq\left|P_{k, l}\left(\tau_{i}, f_{j}\right)\right|_{<_{H_{0}}}^{>_{H_{1}}^{H_{1}}} \Lambda_{i,}
$$

where $\mathrm{H}_{1}$ and $\mathrm{H}_{0}$ are the "target present" and "target absent" hypotheses, respectively, while $\mathrm{P}_{\mathrm{k}, \mathrm{l}}\left(\tau_{\mathrm{i}}, \mathrm{f}_{\mathrm{j}}\right)$ is the complex bistatic power spectral density (PSD). The detection threshold $\Lambda_{\mathrm{i}, \mathrm{j}}=\mathrm{T}_{\mathrm{CA}} \cdot \mathrm{Z}_{\mathrm{i}, \mathrm{j}}$ is given by the product of a deterministic constant scale factor $\mathrm{T}_{\mathrm{CA}}$, which depends on the desired constant false alarm probability $\mathrm{P}_{\mathrm{FA}}$, and the noise power estimate $\mathrm{Z}_{\mathrm{i}, \mathrm{j}}[12]$. Then, the time delays corresponding to all the positive detections are transformed into Cartesian coordinates defining the target positions, which translate into isorange areas defined by ellipsoidal crowns [13],[16].

When individual bistatic range values are recorded, a common method to estimate target position is "multilateration" [13]. Given a single target illuminated by all the $\mathrm{M} \times \mathrm{N}$ bistatic radar pairs, the area of intersection common to the set of decentralized range measurements represents the "uncertainty" region in which the target could possibly insist. An "AND" decision rule (i.e., a target is declared present when it is correctly detected by all the $\mathrm{M} \times$ $\mathrm{N}$ channels) provides the smallest enclosed area among the isorange curves intersections. Range sidelobes can be reduced by applying an appropriate filtering window (e.g., a Hamming window) to the matched filter. However, this choice broadens the response of the main lobe, thus leading to less resolved targets in range, increasing the amount of cross-intersection among isorange ellipsoidal crowns [13].

\section{B. Image Mode: Coherent MIMO processing}

The proposed photonic architecture of the radar network assures an excellent phase coherence. In fact, as reported in Fig. 1, the same optical clock is used first in transmission for the up-conversion and then at the receiver for the downconversion, for all the radar signals. Moreover, the optical oscillator stands out for its low phase noise (PN) curve. As reported in [17], if the angular jitter of the architecture is lower than $10^{-1} \mathrm{rad}$, the effects on MIMO coherent detection performance are negligible. The temporal jitter of the considered system architecture (i.e., the integration of the oscillator PN for offset frequencies of interest $[20 \mathrm{~Hz}, 200$ $\mathrm{MHz}]$ ) is in the order of $10^{-12} \mathrm{~s}$, while the angular jitter is in the order of $10^{-2}$ rad, far better than the limit identified in [17]. For this reason, a coherent MIMO processing can be examined.

Under the ideal assumption that neither amplitude noise nor phase noise is present, the signal received by the $l^{\text {th }}$ receiver, as defined in [18], is equal to:

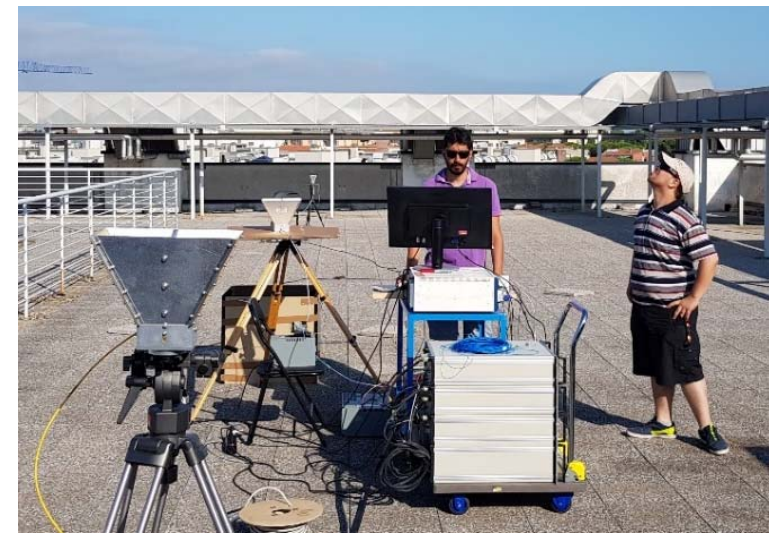

Fig. 3: Picture taken during the measurement campaign.

$$
r_{k, l}(t)=a \cdot s_{k}\left(t-\tau_{k, l}(x, y)\right)
$$

where $s_{k}$ is the signal transmitted by the $k^{\text {th }}$ transmitter, and $a$ is an amplitude factor, while $\tau_{k, l}(x, y)$ is the time delay between the transmitted and received signal and it depends only on the location $(x, y)$ in the Cartesian plane of the target and on the $k^{\text {th }}$ transmitter and $l^{t h}$ receiver. If we consider also amplitude and phase noise contributions, eq. (2) can be recast as follows:

$$
\begin{gathered}
r_{k, l}(t)=a \cdot s_{k}(t- \\
\left.+\tau_{k, l}(x, y)\right) e^{j\left[\theta\left(t-\tau_{k, l}(x, y)\right)-\theta(t)\right]} \\
+n_{k, l}(t)
\end{gathered}
$$

where $n_{k, l}(t)$ represents the amplitude noise term, which is generally modelled as an additive white Gaussian noise. The term $\theta(t)$ takes into account the phase shift caused by the oscillator instability (i.e., the angular jitter obtained integrating the phase noise introduced by the oscillator on the spectral window of interest). If the angular jitter is below the $0.1 \mathrm{rad}$ limit, the phase noise influence on the received signal is negligible. Therefore, the following log-likelihood function suitable for a MIMO approach can be adopted:

$$
\begin{gathered}
\ln [\mathrm{f}(\mathrm{r}(\mathrm{t}) \mid(\mathrm{x}, \mathrm{y}))]=\mathrm{c} \cdot \mid \sum_{\mathrm{k}=1}^{\mathrm{M}} \sum_{\mathrm{l}=1}^{\mathrm{N}} \mathrm{e}^{-\mathrm{j} 2 \pi \mathrm{f}_{\mathrm{c}} \tau_{\mathrm{k}, \mathrm{l}}(\mathrm{x}, \mathrm{y})} . \\
\int \mathrm{r}_{\mathrm{k}, \mathrm{l}}^{\mathrm{b} *}(\mathrm{t}) \cdot \mathrm{s}_{\mathrm{k}}^{\mathrm{b}}\left(\mathrm{t}-\tau_{\mathrm{k}, \mathrm{l}}(\mathrm{x}, \mathrm{y})\right) \mathrm{dt} \mid+\mathrm{c}^{\prime \prime} .
\end{gathered}
$$

According to eq. (4), for each possible target location with coordinates $(x, y)$ the decision statistic is computed determining for all the $M$ transmitters and $N$ receivers the correlation between the received and transmitted signal at baseband. Moreover, a phase term compensates the phase shift due to the time delay $\tau_{k, l}(x, y)$ and to the considered transmitter-receiver pair. After re-phasing each term, all $M x N$ correlation contributes are summed together coherently. For further details (i.e., for constants c' and c'"), see eq. 6 in [18].

It is worth to note that, if the received signal suffers a nonnegligible random phase shift (e.g., in the case of a different system architecture with multiple oscillators, or of an oscillator with worse characteristics in terms of $\mathrm{PN}$ ), the sum in eq. (4) cannot be maximized through compensation of the phase shift due to the time delay $\tau_{\mathrm{k}, \mathrm{l}}$. Indeed, in such an unfavorable condition vector re-phasing would be useless and a non-coherent processing is the only option.

\section{EXPERIMENTAL SETUP}

The proposed coherent radar system is tested in an outdoor, open-field scenario, organized on the rooftop of our lab. The coherent photonic radar network is deployed, as 

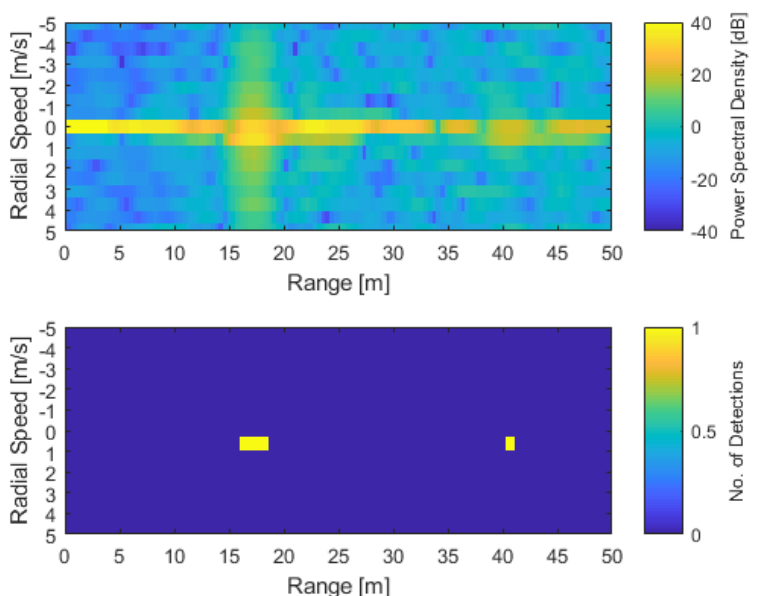

Fig. 4: Range-Radial speed map of channel 1 (TX1-RX1) before MTI filtering (top); CA-CFAR detection of channel 1 (TX1-RX1) after MTI filtering (bottom);

depicted in Fig. 2, with the RX and TX antennas aligned over a $21 \mathrm{~m}$-long baseline. These are oriented upwards, in order to mitigate clutter and multipath returns due to surrounding structures, buildings and vegetation. The antennas are tilted to ensure the simultaneous illumination of the target. The target is a cylinder made of a tight-mesh metal net, hanging from a small drone moving along the baseline. The target height $\mathrm{H}$ is always kept between 15 and $20 \mathrm{~m}$, i.e., where the antenna radiation patterns surely overlap. The drone movements are witnessed by the velocity of the target extracted thanks to the Doppler effect. The output power from each antenna is 20 $\mathrm{dBm}$ (i.e., $100 \mathrm{~mW}$ ). Fig. 3 shows a picture taken during the measurement campaign.

\section{RESUlTS}

In this Section, the target detection and localization capabilities of the photonics-based coherent radar network are presented and discussed. It is worth to remark that the two proposed processing modes are not alternative; the "Search Mode" is preparatory to the application of the "Image Mode", which is intended to provide superior target localization.

\section{A. Search Mode: Decentralized non-coherent processing}

The signals from the two RXs are acquired by the ADCs at $400 \mathrm{MS} / \mathrm{s}$. Each stream is filtered with an ideal BPF having a BW of $100 \mathrm{MHz}$ centred at the IF of $100 \mathrm{MHz}$. Then, the signal is down-converted to $\mathrm{BB}$ and the 4 channels are extracted ( 2 for each RX). Range-Doppler PSD is evaluated on a 25-ms observation time, corresponding to 500 PRIs.

In Fig. 4 (top), the range/radial speed PSD is depicted for the channel TX1-RX1. The graph shows a strong stationary clutter, which can anyway be suppressed removing the zero Doppler frequency. Besides this, the target is clearly visible at about $18 \mathrm{~m}$ with a radial speed slightly less than $1 \mathrm{~m} / \mathrm{s}$. The associated radial speed components extending between $-5 \mathrm{~m} / \mathrm{s}$ and $5 \mathrm{~m} / \mathrm{s}$ are probably due to the rotating movement of the drone propellers. Unfortunately, another moving object in the scene is also detected at approximately $40 \mathrm{~m}$, which is probably a metal structure (e.g., a vertical pole or a large metal panel) present on the rooftop and moved by the wind.

After MTI filtering, CA-CFAR detection is applied to the PSDs at each channel, with the following parameters: $K_{\tau}=$ $16, K_{\dot{f}}=8, P_{F A}=10^{-5}$, where $K_{\tau}$ and $K_{\dot{f}}$ are the number of training cells along range (i.e. retrieved from the delay) and radial speed (i.e. retrieved from the Doppler frequency), respectively, while the number of guard cells remains unvaried on both dimensions $\left(N_{G, \tau}=N_{G, f}=4\right)$. In Fig. 4 (bottom), the detection map after CA-CFAR is shown. The detector identifies the target correctly with an ambiguity of about $2 \mathrm{~m}$ (bi-static target range $16.5 \mathrm{~m} \div 18.75 \mathrm{~m}$ ) and also detects the moving object present in the scene at approximately $40 \mathrm{~m}$.

Combining the detection maps for all the channels, it is possible to evaluate the range/cross-range cumulative detection map, as depicted in Fig. 5, in which the ellipsoidal crowns represent the isorange regions of positive detections at each channel. The region where all isorange ellipses overlap extends for about $3 \times 3 \mathrm{~m}$ around the central point $\mathrm{T}$ of coordinates $(-12 \mathrm{~m}, 16.5 \mathrm{~m})$. After the data acquisition, the Global Positioning System (GPS) data are extracted from the drone log-file. They are reported in Fig. 5 as red circles, showing that the area of intersection is compatible with the actual target position.

\section{B. Image Mode: Coherent MIMO processing}

The non-coherent search mode procedure has spotted the target in an area centred in $T$ with an ambiguity of $3 \mathrm{~m}$ in range and $3 \mathrm{~m}$ in cross-range. Therefore, Image Mode can focus on a slightly larger area of $5 \mathrm{~m}$ x $5 \mathrm{~m}$ with centre in $\mathrm{T}$. In order to limit the noise and interferences for each channel, the same signal conditioning/pre-processing used in Search Mode has been implemented here as well, except Doppler processing and MTI filtering. Then, the decision statistic in Eq. (4) has been calculated on $161 \times 16$ samples spaced by one wavelength in cross-range and ten wavelengths in range. In Fig. 6 (top), the range/cross-range map (ambiguity function) is shown. Applying a detection threshold on the ambiguity function, as it is evident in Fig. 6 (bottom), the target is correctly detected in an area lying between $[-1.5 \mathrm{~m},-1 \mathrm{~m}]$ in range and $[-0.5 \mathrm{~m}$, $0 \mathrm{~m}$ ] in cross-range, corresponding in the 2-D reference system of Fig. 5 to an area between $[15 \mathrm{~m}, 15.5 \mathrm{~m}]$ in height (y-axis), and [-12.5 $\mathrm{m},-12 \mathrm{~m}]$ along the baseline (x-axis). It is worth underlining that the metallic cylinder used as the target had a radius of $10 \mathrm{~cm}$ and a length of $30 \mathrm{~cm}$ approximately. This can explain the detection of 2 points spaced $30 \mathrm{~cm}$ in cross-range. The high level of the detection side lobes in Fig. 6 (top) is due to the small MIMO configuration (only $2 \times 2$ ) used in the experiment: a larger number of TXs and RXs would significantly reduce them.

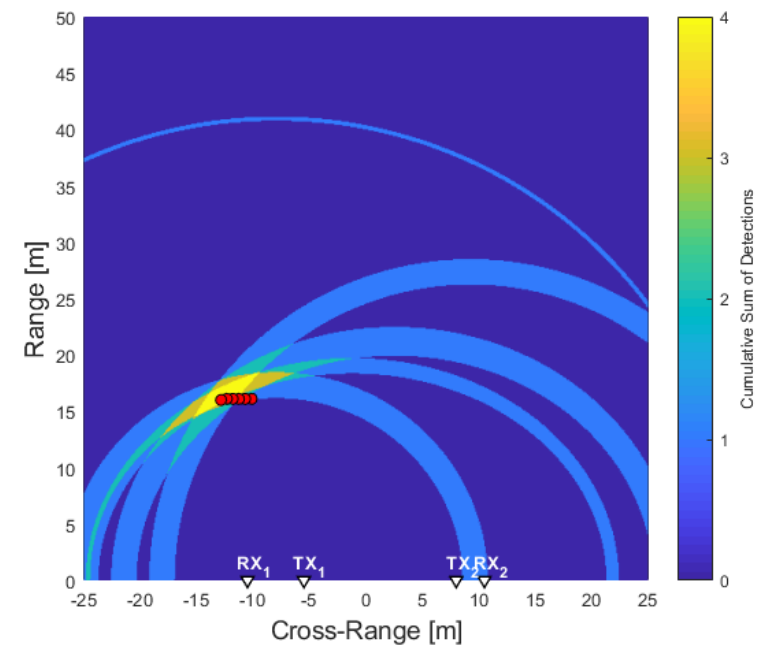

Fig. 5: Search Mode with decentralized non-coherent processing: cumulative sum of the CA-CFAR detections retrieved at each TX-RX bistatic radar pair. Drone positions (red circles) are superimposed in the range/cross-range map. 

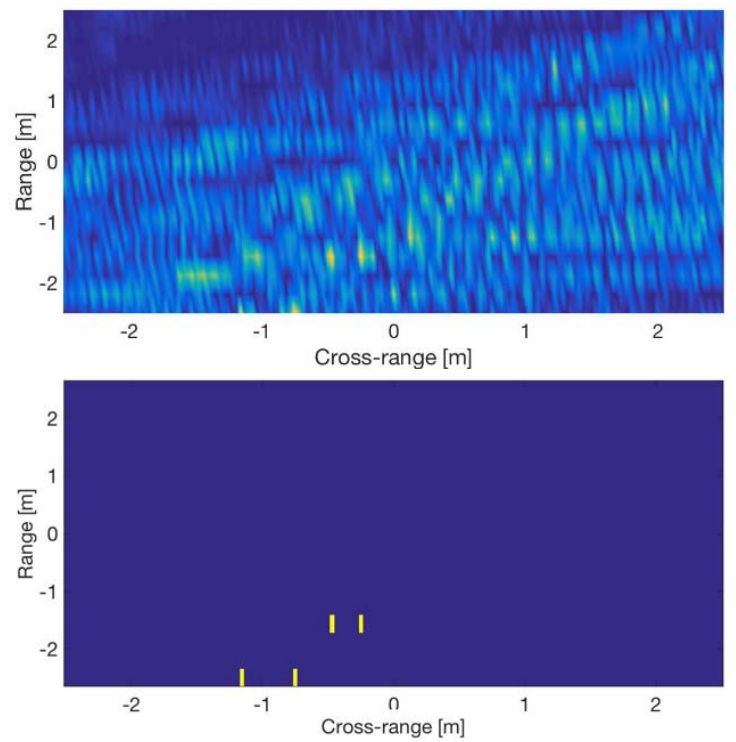

Fig. 6: Image Mode: Range/Cross-range map (top); detection map (bottom);

The reported experiment is realized with a scaled-down geometry, therefore the distance between the four antennas would allow an easier configuration based on a central node for data fusion. However, the presence of a long spool of fiber (the ODL in Fig. 1) emulates a distance of $1 \mathrm{~km}$ between the RHs, justifying the analysis as a distributed radar network. Moreover, in such a largely distributed scenario, considering a standard multistatic system where each node performs a local processing and then sends its map to a master node, only 2 channels would be exploited for detection. Therefore, the result from the Image Mode should be compared with the equivalent multistatic configuration, rather than with the outcome of the Search Mode. This means that only the 2 bistatic radar combinations formed by TX1-RX1 and TX2-RX2 would be processed locally by $\mathrm{RH} 1$ and $\mathrm{RH} 2$, and then sent to a central core for data fusion. Consequently, the multilateration data fusion would have only 2 channels instead of 4 , resulting in a detection ambiguity higher than the one obtained with our non-coherent Search Mode, deriving from the interception of only two elliptical crowns.

Therefore, given the reduced MIMO configuration and the non-idealities introduced by the real environment such as clutter, interferences and target RCS fluctuations, the result obtained in terms of ambiguity reduction is remarkable.

\section{CONCLUSIONS}

In this paper, for the first time to our knowledge, a photonics-based 2x2 MIMO radar network has been tested in a real environment in presence of clutter, interfering signals and other phenomena that impact on the detection, including fluctuating RCS and multipath fading. By means of a standard radar processing technique, the target has been correctly detected in Search Mode with a precision of $2 \mathrm{~m}$ in both range and cross-range. Then, implementing an Image Mode based on a coherent MIMO processing, the detection precision has been increased by a factor 20 , reaching approximately $10 \mathrm{~cm}$ in both dimensions.

The results obtained in this MIMO coherent radar network experiment confirm that photonics is an enabling technology for MIMO radar networks with widely separated antennas. The unsolved issues of the RF domain, such as the signal distribution and distortion on long distances, are straightforwardly solved in the optical domain, and the orthogonality between the transmitted signals can be easily achieved by means of time multiplexing.

The ambition of this scaled-down experiment is mainly to demonstrate the feasibility of the distributed coherent MIMO approach based on photonics. Additional field trials are planned, using more transmitters and receivers and with multiple targets, to further prove the resolution capability of such radar network with widely separated antennas.

\section{ACKNOWLEDGMENTS}

This work has been partially supported by the EU project ROBORDER (\#641388) and NATO SPS SOLE project.

\section{REFERENCES}

[1] T. Mizuno, Y. Miyamoto, "High capacity dense space division multiplexing transmission”, Optical Fiber Technology, vol.35, pp. 108117, February 2017.

[2] A.M. Haimovich et al, "MIMO radar with widely separated antennas", IEEE Signal Processing Magazine vol.25, pp. 116-129, January 2008.

[3] P. Ghelfi et al, "A fully photonics-based coherent radar system", Nature, Vol 507, March 2014.

[4] F. Laghezza et al, "Field Evaluation of a photonics-based radar system in a maritime environment compared to a reference commercial sensor", IET Radar Sonar \& Navigation Journal, Vol 9, n. 8, 2015.

[5] F. Zhang, B. Gao, S. Pan, "Photonics-based MIMO radar with highresolution and fast detection capability", Optics Express, 26, 13, pp. 17529-17540, 2018

[6] L. Lembo et al, "Analysis of a Coherent Distributed MIMO PhotonicsBased Radar Network”, European Radar Conference (EuRAD 2018), pp. 170-173, September 2018.

[7] J. Fu, S. Pan, "A fiber-distributed bistatic ultra-wideband radar based on optical time division multiplexing", International Topical Meeting on Microwave Photonics (MWP), pp. 1-4, October 2015.

[8] G. Serafino, P. Ghelfi, P. Perez-Millan et al., "Phase and amplitude stability of EHF-band radar carriers generated from an active modelocked laser,” J. Lightwave Technol., vol. 29, no. 23, Dec. 2011.

[9] P. Ghelfi, F. Scotti, et al, "Novel architecture for a photonics-assisted radar transceiver based on a single mode-locking laser", IEEE Photon. Technol. Lett., 23 (10), 2011, pp. 639-641

[10] P. A. Williams, W. C. Swann, and N. R. Newbury, "High-stability transfer of an optical frequency over long fiber-optic links," J. Opt. Soc. Am. B 25, 1284-1293 (2008)

[11] F. Laghezza, F. Scotti, P. Ghelfi, A. Bogoni and S. Pinna, "Jitter-limited photonic analog-to-digital converter with 7 effective bits for wideband radar applications," 2013 IEEE Radar Conference (RadarCon13), Ottawa, ON, 2013, pp. 1-5.

[12] G.A. Richards, "Fundamentals of Radar Signal Processing", 2nd Ed., McGraw-Hill, 2014.

[13] S.R. Doughty, "Development and performance evaluation of a multistatic radar system," Doctoral Thesis, Univ. of London, 2008.

[14] P.P. Gandhi, and S.A. Kassam, "Analysis of CFAR Processors in Nonhomogeneous Background," IEEE Transactions on Aerospace and Electronic Systems, vol. 24, pp. 427-445, July 1988.

[15] D.K Barton., A.L. Sergey, Radar Technology Encyclopedia, Artech House, London 1997.

[16] N.J. Willis, Bistatic Radar, $2^{\text {nd }}$ Ed. Scitech Publishing, 2005.

[17] I. Pasya et al, "Detection Performance of M-Sequence-Based MIMO Radar Systems Considering Phase Jitter Effects", IEEE Phased Array Systems \& Technology, 2013.

[18] N.H. Lehmann et al, "High Resolution Capabilities of MIMO Radar", Fortieth Asilomar Conference on Signals, System and Computers, pp. 25-30, October 2006. 